\title{
Novel Thiol-Ene Hybrid Coating for Metal Protection
}

\author{
Mona Taghaviksih ${ }^{1}$, Surya Subianto ${ }^{1}$, Naba Kumar Dutta ${ }^{1,2, *}$ and Namita Roy Choudhury ${ }^{1,2, *}$ \\ 1 Future Industries Institute, University of South Australia, Mawson Lakes Campus, Mawson Lakes, Adelaide, \\ SA 5095, Australia; mona.taghavikish@mymail.unisa.edu.au (M.T.); surya.subianto@unisa.edu.au (S.S.) \\ 2 School of Chemical Engineering, The University of Adelaide, Adelaide, SA 5005, Australia \\ * Correspondence: namita.choudhury@unisa.edu.au (N.K.D.); naba.dutta@unisa.edu.au (N.R.C.); \\ Tel.: +61-883-023-719 (N.K.D.); +61-883-023-546 (N.R.C.)
}

Academic Editor: Alessandro Lavacchi

Received: 13 January 2016; Accepted: 25 March 2016; Published: 7 April 2016

\begin{abstract}
A novel hybrid anticorrosion coating with dual network of inorganic ( $\mathrm{Si}-\mathrm{O}-\mathrm{Si}$ ) and organic bonds (C-S-C) was prepared on metal through an in situ sol-gel and thiol-ene click reaction. This novel interfacial thin film coating incorporates (3-mercaptopropyl) trimethoxysilane (MPTS) and 1,4-di(vinylimidazolium) butane bisbromide based polymerizable ionic liquid (PIL) to form a thiol-ene based photo-polymerized film, which on subsequent sol-gel reaction forms a thin hybrid interfacial layer on metal surface. On top of this PIL hybrid film, a self-assembled nanophase particle (SNAP) coating was employed to prepare a multilayer thin film coating for better corrosion protection and barrier performance. The novel PIL hybrid film was characterised for structure and properties using Fourier transform infrared spectroscopy (FTIR), differential scanning calorimetry (DSC), and thermogravimetric analysis (TGA). The corrosion protection performance of the multilayer coating was examined using potentiodynamic polarization and electrochemical impedance spectroscopy (EIS). The results reveal that this novel double layer coating on metal offers excellent protection against corrosion and has remarkably improved the barrier effect of the coating.
\end{abstract}

Keywords: hybrid film; (3-mercaptopropyl)trimethoxysilane (MPTS); polymerizable ionic liquid (PIL); thiol-ene reaction

\section{Introduction}

Surface polymerisation offers great potential for surface functionalization of various substrates for biomedical, barrier, and anticorrosion applications. Amongst various polymerisation routes, thiol-ene click chemistry offers a facile route to cross-linked polymer network prepared via thiol-ene reaction and is very popular for developing thin films and coatings in biomedical application. On the other hand, sol-gel derived thin film coatings are an excellent candidate for environmentally compliant alternatives for conventional toxic chromate coatings [1-3]. Over the past 30 years, sol-gel chemistry has emerged as a promising approach for a wide range of thin film applications due to its advantages including chemical inertness, room temperature synthesis, commercially demonstrated scale-up potential and good adhesion to both metallic substrates and organic top coats [4,5]. An excellent adhesion of sol-gel films to metals derives from the formation of strong covalent $\mathrm{Si}-\mathrm{O}-\mathrm{M}$ bonds [6]. However, inorganic sol-gel derived films cannot provide adequate barrier properties to prevent the aggressive environmental species to interact with the surface due to their brittle nature and the pores and cracks resulting during the drying process. Incorporation of an organic component to the sol-gel systems leads to the formation of more flexible and functionalized films with improved protective ability [7]. Therefore, the synergistic combination of inorganic and organic materials is ideal for the development of hybrid materials; where two different phases with complementary physical and chemical properties can be combined [8]. However, the resultant properties of such hybrid materials 
depend not only on the properties of the individual components, but also on the synergy between these components. Thus, control of the structures of the organic and inorganic components and their degrees of organization and interactions are crucial parameters for tailored properties.

In principle, the hybrid inorganic-organic sol-gel coating [9] can be prepared by two ways: (i) the organic and inorganic components are linked through strong chemical bonds or (ii) the organic and inorganic component can be merely embedded into each other without any strong interaction [5]. One facile method for the fabrication of these sol-gel coatings is the Self-assembled Nanoparticle Phase (SNAP) method developed by Donley et al. [10]. The SNAP coating process involves selection of precursor compounds based on the desired functionality, formation of "particle-like" nano-sized functionalized siloxane oligomers in situ in an aqueous-based high water content solution; and subsequently crosslinking the siloxane oligomers upon application to form a thin, dense film [11]. While SNAP coatings have certain inherent advantages, however, often their usages are limited by their brittleness and the presence of micro-cracks and defects. On the other hand, organic coatings can be easily formulated, containing chemical substances such as binders or additives with highly cross-linkable molecules. They can form a dense coating with better toughness and elastic properties. But these coatings are often mechanically weak, display poor wetting and adhesion properties to the polar substrate and poor thermal resistance. To overcome the limitations, a new class of hybrid coating is proposed using a molecular level combination of organic and inorganic components. A new approach in the design of such hybrid material of superior characteristics is to use ionic liquids (ILs). The ILs are liquid-phase organic salts (i.e., ionic compounds) with negligible vapor pressure, high thermal stability, and intrinsic solubility for certain gases. Also room temperature IL (RTIL) derivatives can be made with polymerizable moiety (PIL) that can be processed to films or for making polymer-based capsules as an active ingredient for self-healing coating. Poly(ionic liquid)s (PILs) stand for a subclass of polyelectrolytes, the repeating unit of which has ionic liquid (IL) character. Recently, PILs have attracted significant attention due to their unique characteristics, and the research scope in PILs has significantly expanded. It is generally accepted that the incorporation of IL moieties into the polymer chain combines some of the unique characters of ILs along with the common features of polymers. The synergistic effect immediately produces a variety of new (multi)functional materials. Recently, cross-linked polymer networks prepared via photo-initiated thiol-ene reaction have gained significant interest for developing flexible films and coatings using such facile protocol. Thiol-ene films are attractive for use in coating science due to their adhesive properties; partially because thio-ether linkages have high affinity for adhesion to a range of substrates, including metals. The high strength of adhesion for a thiol-ene film to a substrate also stems from the minimized build-up of stress in the film network. This lower network stress is a result of both the step-growth polymerization mechanism and the delayed onset of gel formation, which are characteristic of crosslinked thiol-ene networks. The choice of (3-mercaptopropyl) trimethoxysilane (MPTS) for click chemistry and as organo-alkoxysilane as precursor to sol-gel coating to improve the protection of metal surface against corrosion is unique. Its particular structure contains both a silane terminal function $\left(-\mathrm{SiO}(\mathrm{Me})_{3}\right)$, known for its grafting abilities on oxidised substrates; and a thiol group $(-\mathrm{SH})$, which usually reacts with metals in their metallic states [12]. The anti-corrosion effect of MPTS on $\mathrm{Cu}$ and Al has been reported [12,13]; however, there is no investigation to employ MPTS based thiol-ene hybrid coating for the preparation of sol-gel coating on steel.

Earlier work shows that under certain conditions the mercapto group can be prone to oxidation [14], which may cause coating degradation. To arrest such degradation and any corrosion, it is necessary to either arrest the cathodic/anodic reaction or insert in the circuit a large resistance, which can impede the movement of ions. Therefore, a multilayer protective coating between the metal and the surrounding is an effective way to arrest these reactions and improve barrier and corrosion performance. Thus, through combining the functionalities of sol-gel, thiol-ene, and PIL chemistry, the aim of our investigation is to create a unique hybrid interfacial network, which would exhibit significant anti-corrosive and barrier performance. The introduction of organo-silane and 
subsequent thiol-ene reaction in the grafted layers was investigated in detail using surface, thermal, and microscopic analysis. On top of this PIL hybrid film, a self-assembled nanophase particle (SNAP) coating was employed to prepare multilayer thin film coatings for better corrosion protection and barrier performance. The novel PIL hybrid film was characterized for chemical structure and properties employing thermogravimetric analysis (TGA), differential scanning calorimetry (DSC), photo-acoustic Fourier-transform infra-red (PA FTIR) study, ${ }^{1} \mathrm{H}$-nuclear magnetic resonance (NMR) and solid state NMR. The corrosion protection performance of the multilayer coating was examined using potentiodynamic polarization and electrochemical impedance spectroscopy (EIS).

\section{Experimental Section}

\subsection{Materials}

1-Vinylimidazole, 3-glycidoxypropyltrimethoxysilane(GPTMS), 1,4-dibromobutane, benzophenone (BP), 3-aminopropyltriethoxysilane, (3-mercaptopropyl) trimethoxysilane (MPTS), tetraethylorthosilicate (TEOS), diethyl ether, acetic acid, and methanol were all obtained from Sigma-Aldrich (Sydney, Australia) and used without further purification Fluorosurfactant FC-4432 was obtained from 3M (Sydney, Australia). Double distilled water (DDW) was used for all experiments.

\subsection{Synthesis of 1,4-di (vinylimidazolium) Butane Bisbromide}

1,4-di (vinylimidazolium) butane bisbromide (DVIMBr) was synthesized by stirring a mixture containing 2:1 molar ratio of 1-vinylimidazole to 1,4-dibromobutane in methanol at $60^{\circ} \mathrm{C}$ for $15 \mathrm{~h}$. After cooling down, the reaction mixture was added drop-wise into $1 \mathrm{~L}$ of diethyl ether. The white precipitate was filtered off, washed, and dried at room temperature until constant weight with a yield of $68 \%$ [15]. ${ }^{1} \mathrm{H}-\mathrm{NMR}\left(300 \mathrm{MHz}, \mathrm{D}_{2} \mathrm{O}, \delta, \mathrm{ppm}\right): 8.98(2 \mathrm{H}), 7.69(2 \mathrm{H}), 7.49(2 \mathrm{H}), 7.04(2 \mathrm{H}), 5.75(2 \mathrm{H})$, $5.33(2 \mathrm{H}), 4.21(4 \mathrm{H}), 1.87(4 \mathrm{H})$.

\subsection{Fabrication of Hybrid Material by Thiol-Ene Reaction}

Organic-inorganic PIL hybrid film was prepared using thiol-ene photo polymerization method with stoichiometric ratio of precursors. In a typical synthesis method, a 2:1 mole ratio of (3-mercaptopropyl) trimethoxysilane (MPTS) ( $1 \mathrm{mmol}, 0.2 \mathrm{~g})$ and DVIMBr $(0.5 \mathrm{mmol}, 0.2 \mathrm{~g})$ was dissolved in the minimum volume of methanol $(0.2 \mathrm{~mL})$ and small amount of $\mathrm{BP}(1 \mathrm{~mol} \%$ of the thiol group). The reaction mixture was stirred for approximately $30 \mathrm{~min}$ in $4 \mathrm{~mL}$ vial while protecting from light and oxygen. The thiol-ene mixture was poured and cast onto a glass slide, and the cast samples were irradiated with a $365 \mathrm{~nm}$ UV lamp (light intensity $=85 \mathrm{~mW} / \mathrm{cm}^{2}$ ) in air for $5 \mathrm{~min}$. This PIL hybrid film has been used for further structural investigation.

\subsection{Preparation of Hybrid Coating on Metal}

The mild steel (Mild Steel CA3SN-G, $0.08 \%$ carbon contents, thickness $0.6 \mathrm{~mm}$ of $1.7 \mathrm{~cm} \times 1.7 \mathrm{~cm}$ ) substrates were sequentially polished using abrasive papers with grit numbers 800,1000 , and 1200 prior to cleaning by sequential immersion for $10 \mathrm{~min}$ each in acetone, hexane, and ethanol solutions to remove surface oils. The substrates were rinsed with distilled water and dried between each solvent wash and after the final solvent wash. Finally, the dried substrate was treated with a $0.4 \mathrm{wt} \%$ solution of $\mathrm{NaOH}$ for $30 \mathrm{~min}$ and rinsed with distilled water, acetone, and dried with nitrogen purge.

The coating solutions with total volume of $20 \mathrm{~mL}$ were prepared by diluting (with methanol) the thiol-ene mixture, which was prepared as discussed above to reach a required volume for dip coating. The final mixtures were vigorously stirred and applied to cleaned metal substrates by dip coating at a withdrawal speed of $10 \mathrm{~cm} / \mathrm{min}$ in a dip coater. The sample was then exposed for 5 min with a UV lamp. After UV irradiation, the solvent evaporated and the organic-inorganic PIL hybrid film formed on the metal surface. At the same time due to the exposure of the solution to methanol, hydrolysis occurred; where $\mathrm{O}-\mathrm{CH}_{3}$ groups were converted to $\mathrm{OH}$ groups. The hydrolyzed silicon alkoxides were 
condensed to form inorganic Si-O-Si networks and simultaneous thiol-ene photo-polymerization of the thiol groups at the other end leading to the formation of highly cross-linked dense networked structure (Figure 1).

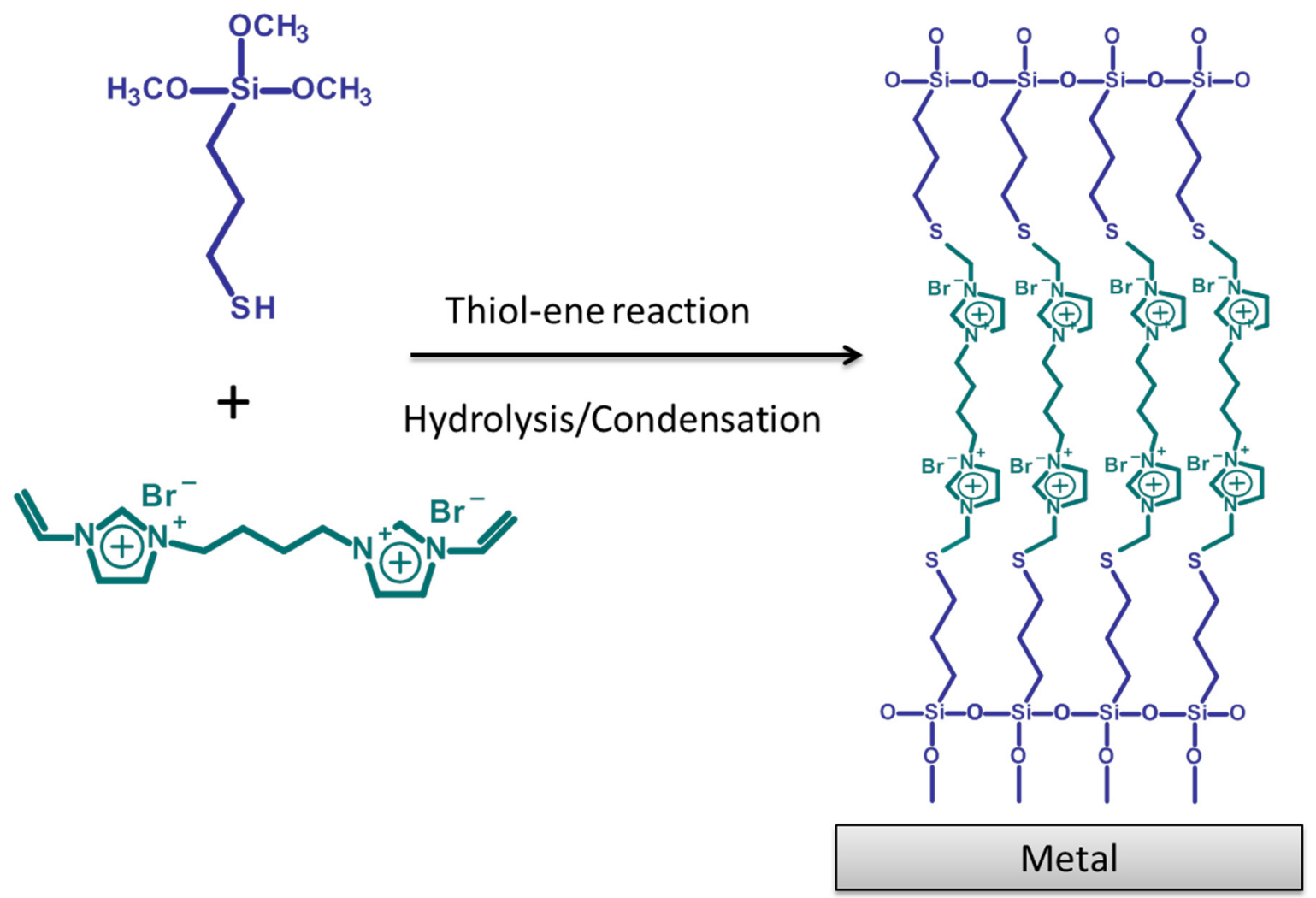

Figure 1. Proposed structure of the MPTS-DVIMBr hybrid film coated on metallic substrate.

\subsection{Preparation of Sol-Gel Coating}

The top layer of the multilayer coating has been produced using the self-assembled nanophase particle (SNAP) as a matrix for coating. In a typical procedure, the SNAP solution was prepared with drop-wise addition of $42 \mathrm{~mL}(0.19 \mathrm{~mol})$ 3-glycidoxypropyltrimethoxysilane (GPTMS) and $13.4 \mathrm{~mL}$ $(0.06 \mathrm{~mol})$ tetraethylorthosilicate (TEOS) to $64.8 \mathrm{~mL}$ solution of acetic acid in doubly distilled water (DDW) under $\mathrm{N}_{2}$. The solution was continuously stirred for $1 \mathrm{~h}$ during addition. The TEOS:GPTMS molar ratio used was (1:3 mol ratio), respectively. Hydrolysis and condensation of silanes were conducted at high water/silane ratio of 15 . After all of the silanes had been added and hydrolyzed, the solution was aged in a closed container for three days under continued stirring at ambient temperature.

The coating solution was prepared by diluting the aged SNAP solution with DDW by a factor of 1.75 and subsequent addition of crosslinking agent (aminosilane) and surfactant (3M FC-4432, $0.04 \mathrm{wt} / \mathrm{v} \%$ ). The final mixture was vigorously stirred and applied to PIL hybrid coated substrates by dip coating at a withdrawal speed of $10 \mathrm{~cm} / \mathrm{min}$. The coating was made within a time frame of 10-30 min, from the point of addition of the crosslinking agent. The coated sample was dried at room temperature for $24 \mathrm{~h}$ to allow solvent evaporation. The coating was thermally cured at $80{ }^{\circ} \mathrm{C}, 60 \%$ humidity overnight in a humidity oven (ESPEC-model SH-241).

\subsection{Characterization}

${ }^{1} \mathrm{H}-\mathrm{NMR}$ analysis was performed on a $300 \mathrm{MHz}$ Bruker Avance spectrometer at room temperature using $\mathrm{D}_{2} \mathrm{O}$ as the solvent. Solid state ${ }^{29} \mathrm{Si}$ CP-MAS NMR measurements were carried out using a Bruker Avance III $400 \mathrm{MHz}$ spectrometer operating at $79 \mathrm{MHz}$ equipped with a $7 \mathrm{~mm}$ probe. Sample was 
spun at $2.5 \mathrm{kHz}$. The spectral width used was $201 \mathrm{ppm}$, the acquisition time employed was $15 \mathrm{~ms}$, and the recycle delay applied was $5 \mathrm{~s}$.

Infrared spectra of the PIL hybrid film were acquired using Nicolet Magna-IR Spectrometer 750 in photo-acoustic mode, 256 numbers of scans, using carbon black as reference.

Differential scanning calorimetry (DSC) measurement was conducted using TA Instruments Discovery DSC. The instrument was calibrated for baseline and cell constant prior to running the experiments. The samples were sealed in hermetic aluminum pans for use in the DSC experiment, and an empty pan was used as the reference. The temperature range for the experiment was $-70{ }^{\circ} \mathrm{C}$ to $200{ }^{\circ} \mathrm{C}$ with a heating rate of $10^{\circ} \mathrm{C} / \mathrm{min}$ under controlled nitrogen gas flow rate of $50 \mathrm{~mL} / \mathrm{min}$. Thermogravimetric analysis (TGA) was performed using a TA Instruments Discovery TGA with an aluminum pan. The experimental samples were subjected to a $10^{\circ} \mathrm{C} / \mathrm{min}$ heating rate from $100{ }^{\circ} \mathrm{C}$ to $700{ }^{\circ} \mathrm{C}$ under a nitrogen atmosphere. The morphology of the coated metal's surface was observed using a scanning electron microscope (SEM, ZEISS Gemini SEM. The samples were placed and images were taken without any further coating at an accelerating voltage of $10 \mathrm{kV}$.

The electrochemical characterization of the uncoated and the coated samples was carried out using a standard corrosion cell (1 L, Pine Research Instrumentation, Durham, NC, USA) with conventional three electrode set-up in non-deaerated $3.5 \mathrm{wt} \% \mathrm{NaCl}$ solution prepared from analytical grade chemicals (ChemSupply, Gillman, Australia) and distilled water (Milli Q). A saturated calomel electrode (SCE, Pine Research Instrumentation, $+0.24 \mathrm{~V}$ vs. Normal Hydrogen Electrode, NHE) was employed as the reference electrode. A graphite rod of $2 \mathrm{~mm}$ diameter was used as the counter electrode and the sample under test was the working electrode. All the reported potentials in this paper (determined using electrochemical techniques) are with reference to SCE. Initially, the open circuit potential (OCP) of the specimens was monitored as a function of time and samples were allowed to stabilize at their OCP for $1 \mathrm{~h}$ before measurements. Low carbon steel coupons (thickness $0.6 \mathrm{~mm}$ ) of $1.7 \mathrm{~cm} \times 1.7 \mathrm{~cm}$ (both coated and uncoated) were used as the samples for electrochemical testing. The sample holder is made of Teflon and a platinum sheet and a platinum wire was used for electrical connection. The area exposed to the electrolyte solution was controlled using inner and outer " $\mathrm{O}$ " rings for an electrode area of $0.785 \mathrm{~cm}^{2}$. All the measurements were carried out at least in duplicates. Potentiodynamic polarization study was conducted at a scan rate of $1 \mathrm{mV} \cdot \mathrm{s}^{-1}$ in the potential range of $\pm 300 \mathrm{mV}$ relative to OCP. The Electrochemical Impedance Spectroscopy (EIS) measurements were performed using a Solartron 1260 impedance/gain-phase analyzer in combination with a Solartron $1280 \mathrm{~B}$ electrochemical interface. The impedance measurements were carried out in a $1 \mathrm{MHz}$ to $10 \mathrm{kHz}$ frequency range with 40 steps per decade. All the spectra were recorded at open circuit potential with applied $10 \mathrm{mV}$ sinusoidal perturbation.

\section{Results and Discussion}

\subsection{Structural Evolution of the PIL Hybrid Film}

FTIR was used to evaluate the final chemical structure of the double network PIL hybrids. The FTIR spectra of MPTS, DVIMBr, and the PIL hybrid film (2:1 ratio) are shown in Figure 2. The bands at $2570 \mathrm{~cm}^{-1}, 1644 \mathrm{~cm}^{-1}$, and $2928-3080 \mathrm{~cm}^{-1}$ that are present in the unreacted MPTS and DVIMBr monomers correspond to the thiol groups, $\mathrm{C}=\mathrm{C}$ stretching of the vinyl group, stretching of $-\mathrm{CH}$ groups of the imidazole ring of the PIL, and vinylic proton $=\mathrm{C}-\mathrm{H}$ stretching, respectively [16]. These bands disappeared in the cured PIL hybrid film indicating the reaction between double bond of vinyl group in PIL, and subsequent network formation through the thiol-ene photo-polymerization.

The FTIR spectrum of MPTS shows a characteristic absorption band at $2850 \mathrm{~cm}^{-1}$ corresponding to $\mathrm{Si}-\mathrm{OCH}_{3}$ group [17]. This characteristic absorption band due to $\mathrm{Si}-\mathrm{OCH}_{3}$ disappeared after PIL hybrid film formation due to hydrolysis of MPTS [18]. During hydrolysis, methoxy groups are converted to $\mathrm{Si}-\mathrm{OH}$ groups and broad peaks at $\sim 910 \mathrm{~cm}^{-1}$ and $\sim 3500 \mathrm{~cm}^{-1}$ appear indicating the presence of $\mathrm{Si}-\mathrm{OH}$ groups in PIL hybrid film [19]. Moreover, the condensation reactions led to the formation of 
Si-O-Si network in the PIL hybrid film. This is evident from the absorption band at $1030 \mathrm{~cm}^{-1}$ [9]. Thus from the FTIR studies, the formation of MPTS-DVIMBr hybrid film through thiol-ene reaction could be inferred.

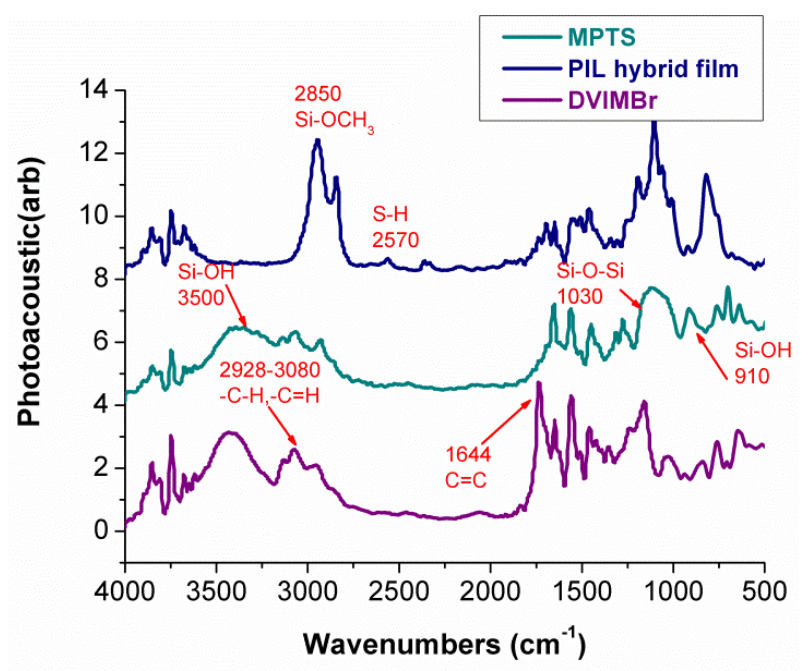

Figure 2. FTIR spectra of MPTS, DVIMBr, and PIL hybrid film.

Scanning electron microscopy (SEM) was employed to investigate film surface morphology. Figure 3 shows the SEM images of the polished steel surface compared to samples coated with SNAP and SNAP/PIL hybrid films. The SEM images show that both the coatings on the metal surface are uniform, defect-free and well adhered to the substrate surface. The comparison of SEM images of bare (Figure 3a) and coated metals (Figure 3b,c) shows that the coatings have fully covered the metal surfaces in homogeneous manner. Although, the addition of the PIL interfacial layer appears to marginally increase the surface roughness; the SNAP/PIL hybrid film still shows a very smooth, uniform surface without cracks or other defects. This observation indicates a uniform distribution of the SNAP particles over the metallic substrate, which would result in a dense, uniform coating.

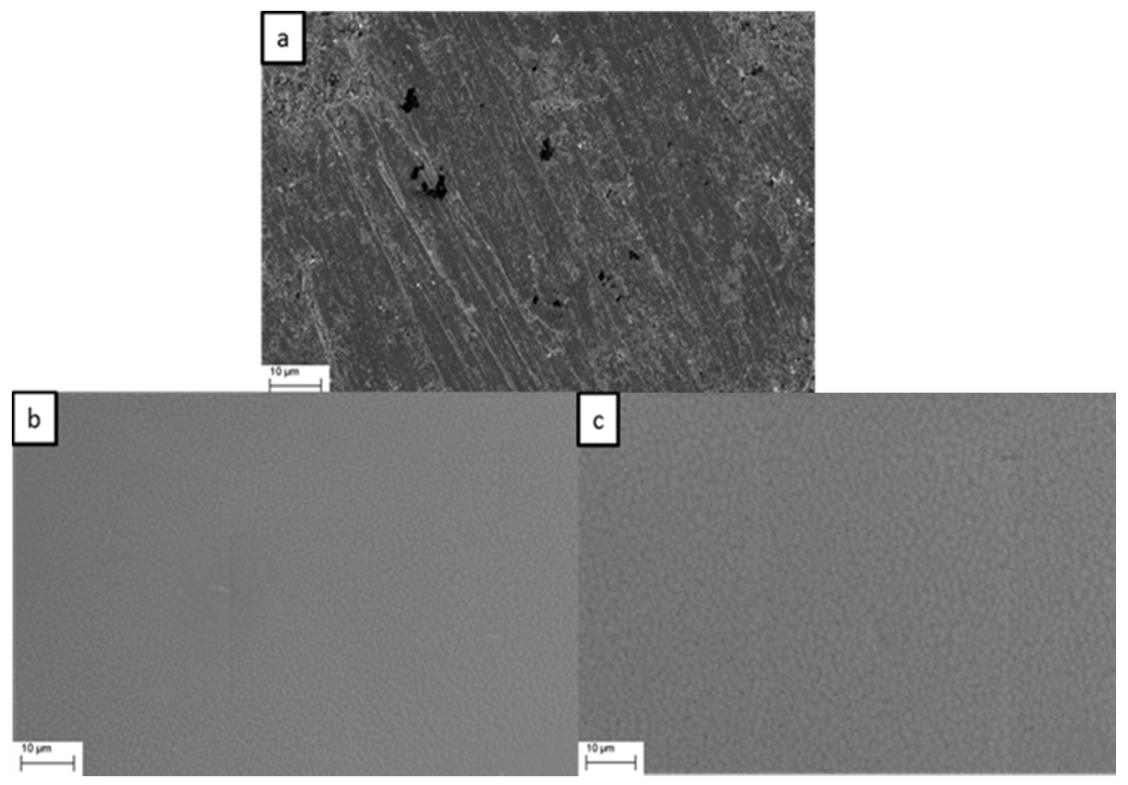

Figure 3. SEM image of (a) polished metal; (b) SNAP coated metal; and (c) SNAP/PIL hybrid film coated metal. 
The solid state CP MAS ${ }^{29} \mathrm{Si}$ NMR of the SNAP/PIL hybrid material also shows that the condensation of the silanes has occurred. The NMR spectrum in Figure 4 shows peaks that can be predominantly attributed to T3 species at -61 and $-69 \mathrm{ppm}$, with smaller peaks at $-37 \mathrm{ppm}$ due to $\mathrm{T} 2$ species, $-101 \mathrm{ppm}$ due to $\mathrm{Q} 3$ and at $-111 \mathrm{ppm}$ due to the presence of $\mathrm{Q} 4$ species [20].

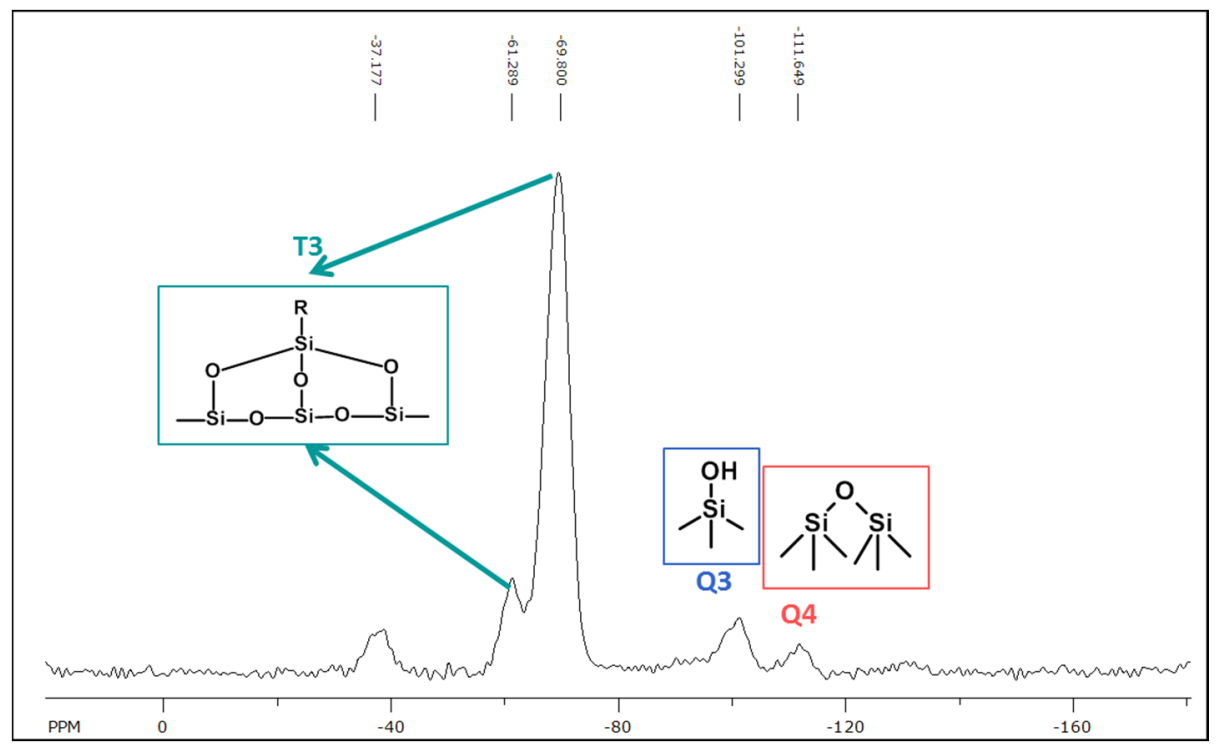

Figure 4. Solid state CP MAS ${ }^{29} \mathrm{Si}$ NMR of the SNAP/PIL hybrid material.

The predominance of the T3 peak is due to the nature of the SNAP nanoparticle, where the main component is GPTMS, where the silica is connected to the glycidol functionality, and thus after condensation the formed SNAP nanoparticles would consist predominantly of silica with T3 structure. In the PIL species the silane used was also mercaptosilane, which can also give rise to a T3 peak when cured and may be the source of the smaller T3 peak at $-61 \mathrm{ppm}$. The presence of the peak at $-37 \mathrm{ppm}$ is likely due to the presence of some incomplete crosslinking and maybe a mixture of $\mathrm{T} 2$ and $\mathrm{T} 1$ species, however based on their respective peak area this is only a small fraction of the material (1:30 ratio of the T2/T1 to T3 peak area). The Q3 species likely arises from the TEOS in the SNAP nanoparticles; which, being only $3 \mathrm{~nm}$ in diameter have a large surface area and thus the Si species would be a mixed of $\mathrm{R}-\mathrm{Si}(\mathrm{O})_{3}$ and $\mathrm{HO}-\mathrm{Si}(\mathrm{O}-\mathrm{Si})_{3}$ as can be expected based on the chemical structure of the SNAP nanoparticle. There is also a small peak due to Q4 species $\left(\mathrm{Si}(\mathrm{OSi})_{4}\right)$, which is likely due to further condensation of the silanes; however this peak represents only a small portion of the bulk material due to the low temperature used in curing these SNAP/PIL hybrid film $\left(80^{\circ} \mathrm{C}\right)$. Q4 species are generally the result of higher temperature thermal treatment.

\subsection{Thermal Stability of the PIL Hybrid Film}

The thermal degradation behavior of the prepared PIL hybrid materials and the monomers were examined using TGA. The TGA curve of MPTS-DVIMBr hybrid film shows one main degradation peak at around $60 \mathrm{wt}$. \% weight loss at onset temperature of $250{ }^{\circ} \mathrm{C}$ and peak degradation temperature of $340{ }^{\circ} \mathrm{C}$ (Figure 5). The TGA also indicates an initial weight loss at $100-250{ }^{\circ} \mathrm{C}$ for PIL hybrid film, which could be attributed to the presence of a trace amount of solvent and unreacted precursor trapped in the PIL hybrid film. The degradation process at high temperature involves the cleavage of C-Si, $\mathrm{Si}-\mathrm{O}-\mathrm{Si}$ and $\mathrm{Si}-\mathrm{OH}$ bonds in the PIL hybrid film, which is almost $10^{\circ} \mathrm{C}$ higher than that of the PIL, and more than $200^{\circ} \mathrm{C}$ higher than MPTS. Thus the results of TGA confirm the thermal stability of the PIL hybrid film, which is attributed to the highly cross-linked network of the hybrid through the formation of $\mathrm{Si}-\mathrm{O}-\mathrm{Si}, \mathrm{Si}-\mathrm{OH}$ networks and an organic network through the thiol-ene photo-polymerization. In order to obtain an estimation of the inorganic content of both the PIL hybrid film and the SNAP/PIL 
hybrid film; TGA was carried out under oxygen up to $900{ }^{\circ} \mathrm{C}$. As it has been illustrated in Figure $5 \mathrm{c}$ PIL film without SNAP contains $22 \%$ residue compared with $43 \%$ for PIL/SNAP hybrid film. This is due to the fact that PIL/SNAP hybrid film contains two major inorganic components plus S, N, and Si elements; and thus can be expected to have more residue compared to PIL film alone. The PIL film alone is more organic in nature as the bulk of the material is the organic PIL, with the residue expected to come from the mercaptosilane end-caps and nitrogen from PIL, whereas in SNAP/PIL hybrid film the SNAP itself is silica-based. Also PIL/SNAP hybrid film shows two degradation temperatures. The second degradation around $600{ }^{\circ} \mathrm{C}$ could be related to the degradation of nonsilica and other oraganic components of SNAP/PIL hybrid film.
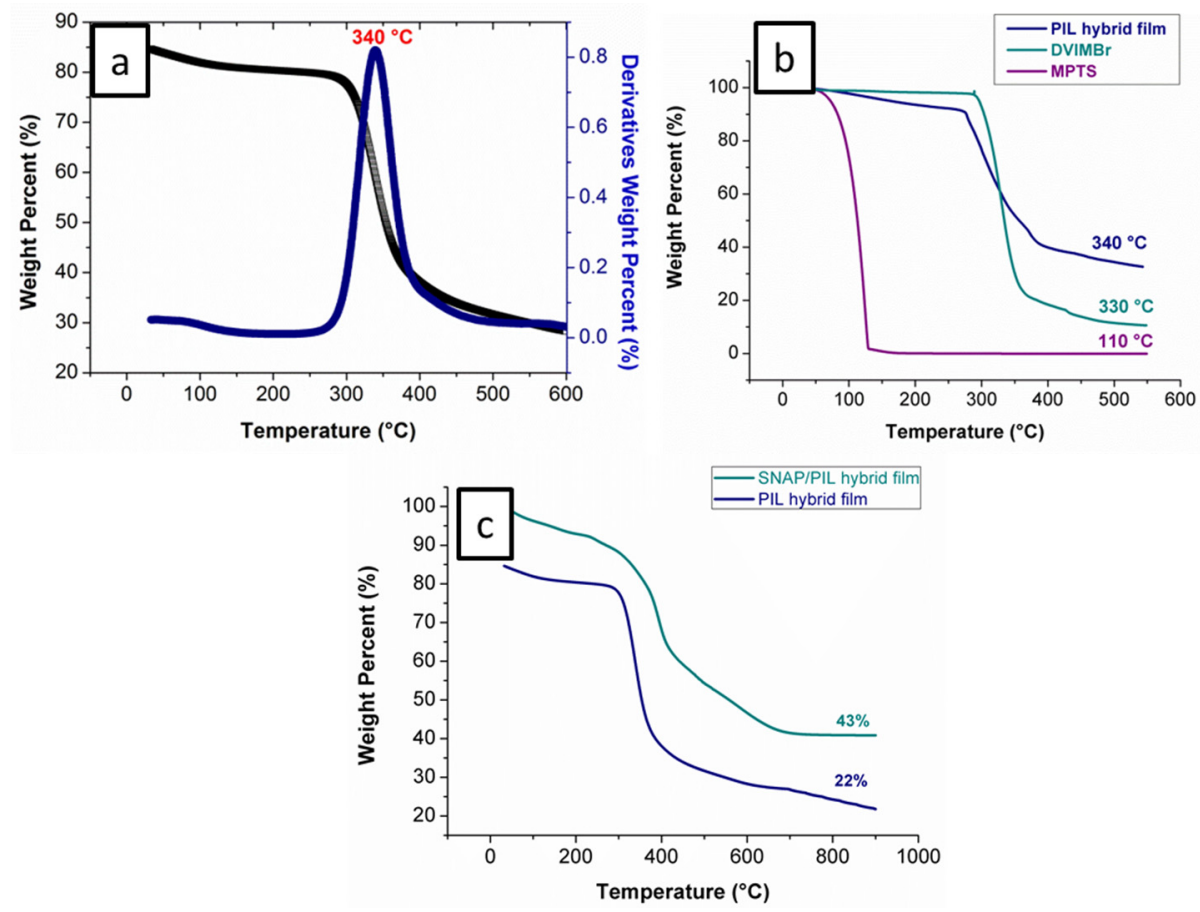

Figure 5. TGA curves of (a) PIL hybrid film with first derivative vs. temperature; (b) degradation behavior of the PIL hybrid film and the precursors; and (c) TGA under oxygen.

The glass transition $\left(T_{\mathrm{g}}\right)$ temperature of the PIL hybrid film is a critical parameter, which correlates to the barrier properties and establishes maximum service temperature of the film, and has been determined from differential scanning calorimetry (DSC). The $T_{\mathrm{g}}$ of the PIL hybrid material is found to be $21^{\circ} \mathrm{C}$, whereas $T_{\mathrm{g}}$ of crosslinked DVIMBr have been observed around $-40{ }^{\circ} \mathrm{C}$ (Figure 6).

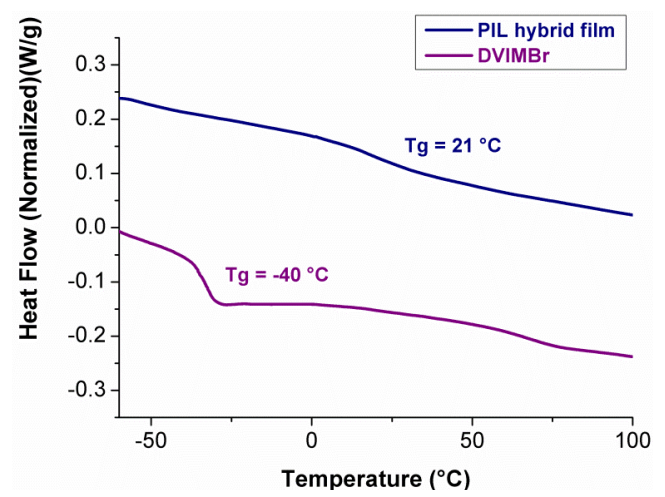

Figure 6. DSC thermogram showing the glass transition temperature, $T_{\mathrm{g}}$ of PIL hybrid film and DVIMBr. 
This positive shift in $T_{\mathrm{g}}$ could be explained by the presence of extensive crosslinking, which limits segmental mobility of the chains and makes a more rigid structure at room temperature. No melting or crystallization peak is observed in DSC curve. The increase in $T_{\mathrm{g}}$ in the PIL hybrid material compared to DVIMBr can be attributed to the presence of extensive network formation as observed from FTIR.

\subsection{Electrochemical Analysis}

Potentiodynamic polarization investigation of the metal coupons coated with SNAP and the PIL/SNAP hybrid film was carried out to determine the electrochemical performance of the PIL hybrid film interfacial layer. Initially, the corrosion potential $\left(E_{c o r r}\right)$ and corrosion current $\left(I_{\text {corr }}\right)$ obtained from the Tafel plot is quite similar between the samples with and without the PIL hybrid interfacial layer; with the SNAP showing a slightly more noble $E_{\text {corr }}$ but with similar $I_{c o r r}$ to the PIL hybrid containing sample (Figure 7). However, after $24 \mathrm{~h}$ immersion in 3.5\% salt solution, there was a significant difference between the two, in both $E_{c o r r}$ and $I_{\text {corr }}$. The values for the PIL/SNAP hybrid film coated samples only changed marginally, especially for $I_{\text {corr }}$, indicating that the coatings have resisted corrosion quite well (Table 1). In contrast, SNAP-only coated samples show a significant increase in $I_{\text {corr }}$ of two orders of magnitude; which indicates a significant increase in the corrosion rate after $24 \mathrm{~h}$ immersion. This observation reveals that, despite the good adhesion between the metal and SNAP, the coating may still allow diffusion of electrolyte thus over time corrosion occurs on the metal surface. The presence of the PIL hybrid film interfacial layer forms a barrier on the metal surface, thus preventing corrosion. The reason can be attributed to several factors; firstly, due to the thiol-ene click reaction, which can improve PIL hybrid film/SNAP coating's interfacial adhesion and crosslinking density. Secondly, the PIL hybrid film interfacial layer may also act as anticorrosion layer as PIL will act as radical scavengers [21]. Thirdly, MPTS improves coating's barrier effect through forming a well-organized organic crosslinking structure $(\mathrm{C}-\mathrm{S}-\mathrm{C})$ and enables the decrease in corrosion of the underlying metal substrate.

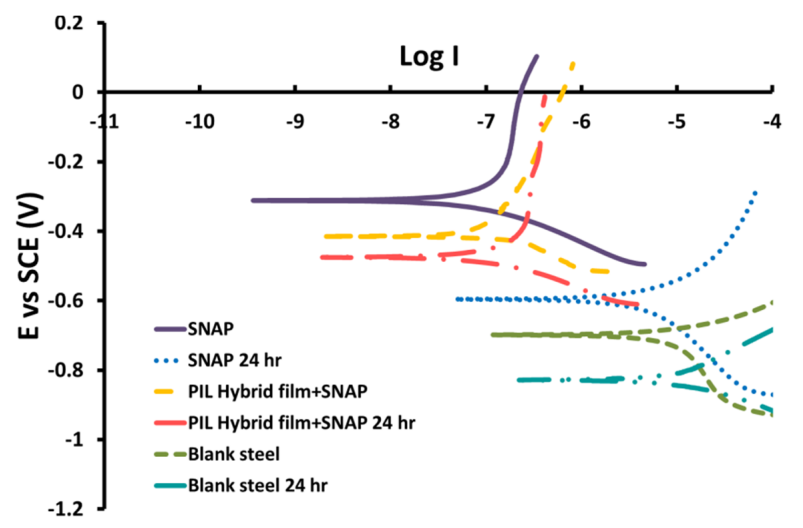

Figure 7. Tafel plot of the SNAP-coated samples with and without the PIL interfacial layer.

Table 1. Corrosion potential $\left(E_{c o r r}\right)$ and current $\left(I_{c o r r}\right)$ of the SNAP-coated samples with and without the PIL hybrid film interfacial layer.

\begin{tabular}{cccc}
\hline Metal Sample & $\boldsymbol{E}_{\text {corr }}(\mathbf{V})$ & $\boldsymbol{I}_{\text {corr }}(\mathbf{A})$ & $\boldsymbol{E}_{\boldsymbol{I}} \mathbf{( \% )}$ \\
\hline Blank metal & -0.674 & $7.9 \times 10^{-6}$ & - \\
SNAP coated & -0.312 & $7.4 \times 10^{-8}$ & - \\
PIL/SNAP Hybrid film coated & -0.395 & $1.3 \times 10^{-7}$ & - \\
Blank metal 24 h & -0.937 & $4.9 \times 10^{-6}$ & - \\
SNAP coated 24 h & -0.613 & $4.1 \times 10^{-6}$ & 16.3 \\
PIL/SNAP Hybrid film coated 24 h & -0.479 & $1.5 \times 10^{-7}$ & 96.9 \\
\hline
\end{tabular}


Moreover, inhibition efficiency $E_{I}(\%)$ is calculated from the values of $I_{c o r r}$ using the following equation [22]:

$$
E_{I}(\%)=\frac{I_{\text {corr }}-I_{\text {corr }}^{\prime}}{I_{\text {corr }}} \times 100
$$

where $I_{\text {corr }}$ and $I^{\prime}$ corr are uninhibited and inhibited corrosion current densities, respectively. The results revealed that the addition of PIL into the SNAP coating increase the $E I$ (\%) from $16.3 \%$ to $96.9 \%$ after $24 \mathrm{~h}$ immersion (Table 1).

This observation was also confirmed with the electrochemical impedance analysis, which shows a significant shift in the phase angle for samples with the PIL/SNAP-coated samples. As shown in Figure $8 \mathrm{~b}$, the impedance modulus is greater for samples coated with PIL/SNAP hybrid film interfacial layer compared to those coated with SNAP alone. The impedance modulus in the bode plot also remained almost unchanged after $24 \mathrm{~h}$ immersion in salt solution, compared to samples coated with only SNAP, where there is an order of magnitude drop in impedance modulus after $24 \mathrm{~h}$. The phase angle shown in Figure 8a also shows significant differences in the samples coated with PIL/SNAP hybrid film, which appears as an additional time-constant at middle frequencies.
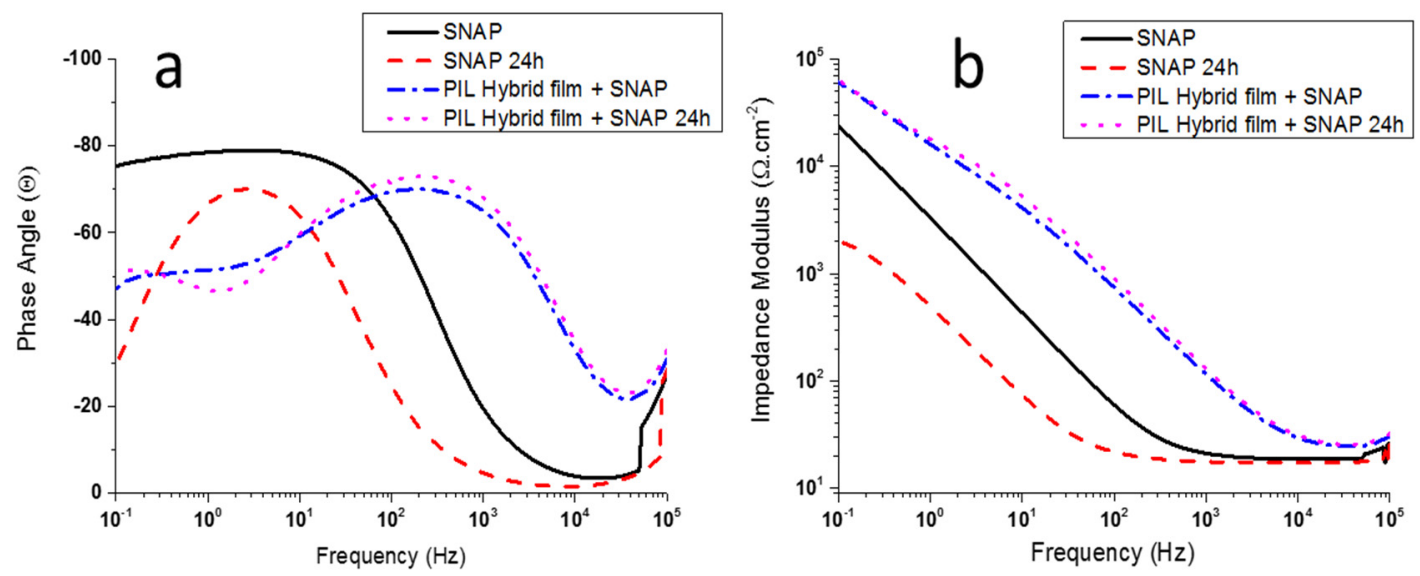

Figure 8. (a) Phase angle; (b) Impedance modulus of the SNAP-coated samples with and without the PIL interfacial layer.

These observations confirm the presence of the interfacial layer (which manifests as an additional capacitance) which changes the coating behavior from resistive to capacitive at these frequencies as it acts as a barrier to charge transfer. Similarly to the impedance modulus, the sample coated with the PIL/SNAP hybrid film also shows very little change after $24 \mathrm{~h}$ immersion in salt solution, indicating that it is structurally unchanged. In contrast, the phase angle of the sample coated only with SNAP coating shows a transition from a capacitor-like behavior to a resistor-like behaviors after $24 \mathrm{~h}$, indicating that it may allow increased charge transport and diffusion.

This observation indicates a greater resistance of the coating, of which the resistance due to interfacial charge transfer and diffusion through the bulk coating can be modelled using an appropriate equivalent circuit (Figure 9). The analyses and fitting of the impedance curves of these samples (Table 2 and Figure 10) show that the presence of the PIL interfacial layer initially reduces the interfacial resistance by an order of magnitude, possibly due to the SNAP only coating forming a denser coating on the interface without the interfacial layer. However, it also significantly increased the coating resistance by several orders of magnitude, indicating that the coating itself becomes less permeable and more resistive compared to the SNAP-only coating as were seen from the bode plots and phase angle diagram. The capacitance values obtained are also initially similar, and was within the expected range for sol-gel coated samples [23]. However, after $24 \mathrm{~h}$ immersion in salt solution there was a significant drop in the interfacial and coating resistance of the SNAP only sample, with a decrease from $1.7 \mathrm{M} \Omega$ to $2.6 \mathrm{k} \Omega$ in interfacial resistance for the SNAP sample while the interfacial resistance of the PIL/SNAP 
hybrid film remains similar to before. This trend indicates that the interface between the metal and SNAP coating has corroded, as the SNAP coating is still permeable to electrolyte. When the PIL hybrid film interfacial layer is present, the interfacial resistance remained high and actually increased to $300 \mathrm{k} \Omega$ after $24 \mathrm{~h}$ immersion, which indicates that the PIL hybrid layer provides a significant barrier protection and passivation of the metal surface. The more stable capacitance values of the PIL/SNAP hybrid film coated samples also indicate that they are more stable and maintained the integrity of the interfacial layer after $24 \mathrm{~h}$ immersion.

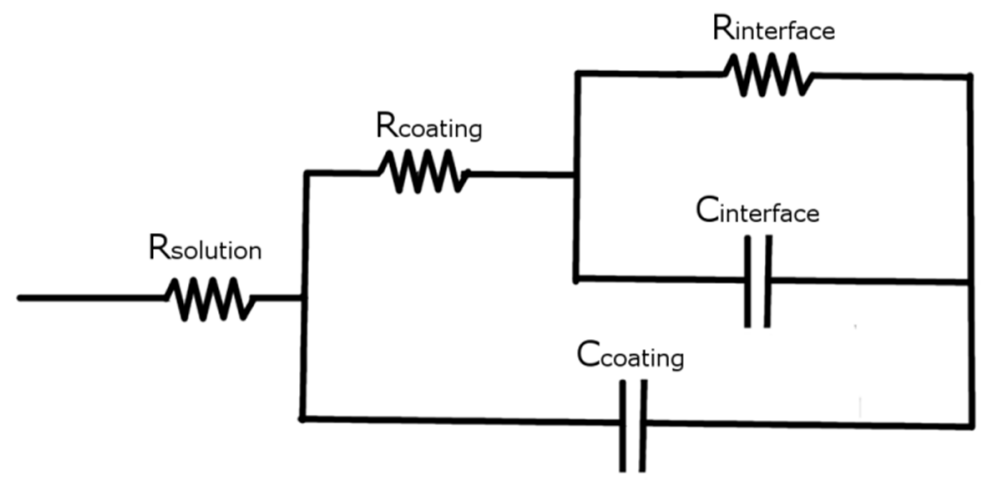

Figure 9. The equivalent circuit used to model the impedance data of a permeable coating.

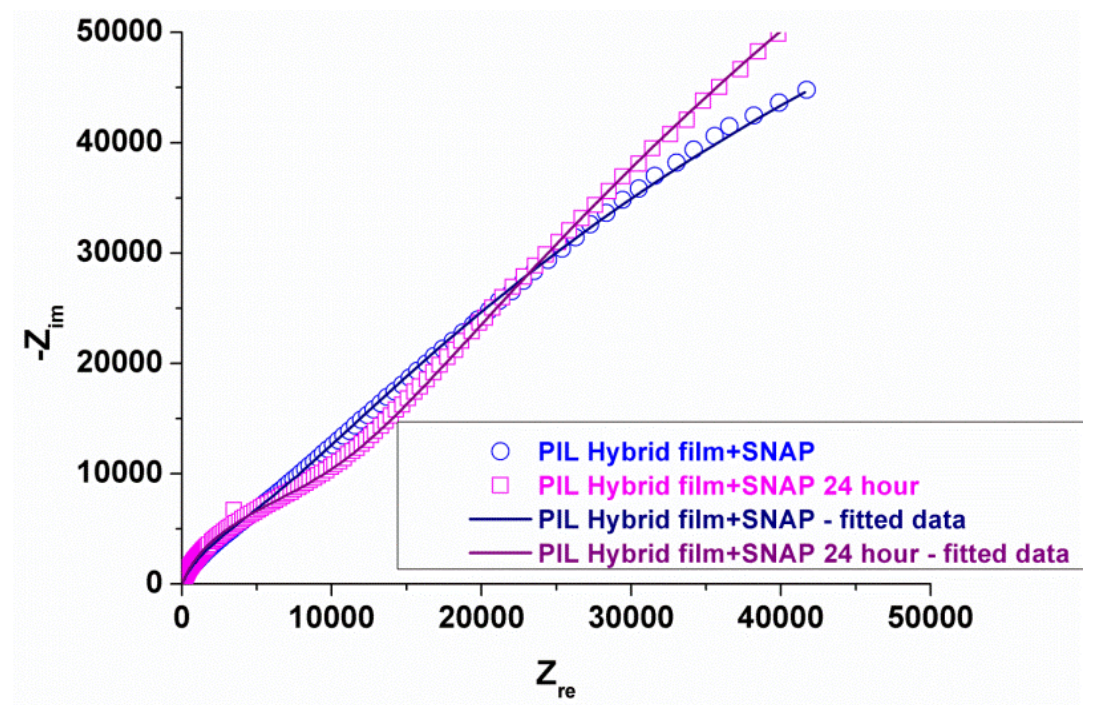

Figure 10. Nyquist plot and modelling result of the impedance data for PIL hybrid film/SNAP coated samples.

Table 2. Resistance and capacitance values obtained from EIS modelling (using the model in Figure 9).

\begin{tabular}{cccccc}
\hline SAMPLE & $\boldsymbol{R}_{\text {solution }}(\Omega)$ & $\boldsymbol{R}_{\text {coating }}(\Omega)$ & $\boldsymbol{C}_{\text {coating }}(\mathrm{F})$ & $\boldsymbol{R}_{\text {interface }}(\Omega)$ & $\boldsymbol{C}_{\text {interface }}(\mathbf{F})$ \\
\hline SNAP only & 21 & 158 & $4.16 \times 10^{-5}$ & $1.17 \times 10^{6}$ & $2.08 \times 10^{-5}$ \\
SNAP only 24h & 12 & 18 & $8.44 \times 10^{-6}$ & 2613 & $4.08 \times 10^{-4}$ \\
PIL/SNAP hybrid film & 23 & 15,257 & $7.04 \times 10^{-6}$ & $1.54 \times 10^{5}$ & $1.44 \times 10^{-5}$ \\
PIL/SNAP hybrid film 24 h & 21 & 15,009 & $4.45 \times 10^{-6}$ & $3.19 \times 10^{5}$ & $1.84 \times 10^{-5}$ \\
\hline
\end{tabular}

Interestingly, the presence of the PIL hybrid layer also seems to impart anti-corrosion properties even in the presence of deliberately-induced defects. When the surface of the sample coated with PIL/SNAP hybrid film was scratched, the impedance spectra (Figure 11a) show a decrease in 
impedance modulus to values similar to that of uncoated metal, which is expected due to the fact that the electrochemical response of the scratched samples would be largely due to the exposed metal surface. However, upon $72 \mathrm{~h}$ immersion in salt solution the impedance spectra of the scratched PIL/SNAP hybrid film coated samples show less change compared to an uncoated metal samples over $3 \mathrm{~h}$. Although the impedance of the sample did not return to values of a fully intact coating, there was a marginal increase in impedance modulus after $12 \mathrm{~h}$; and over the $72 \mathrm{~h}$ duration there was a slow shift in the phase angle towards middle frequencies as was observed with uncoated metal samples, although after $72 \mathrm{~h}$ the shift was less than the uncoated metal after $3 \mathrm{~h}$ (Figure 11b). This observation indicates that the PIL hybrid film acts as an active, anti-corrosion layer, and has the potential to impede corrosion in the defect area.
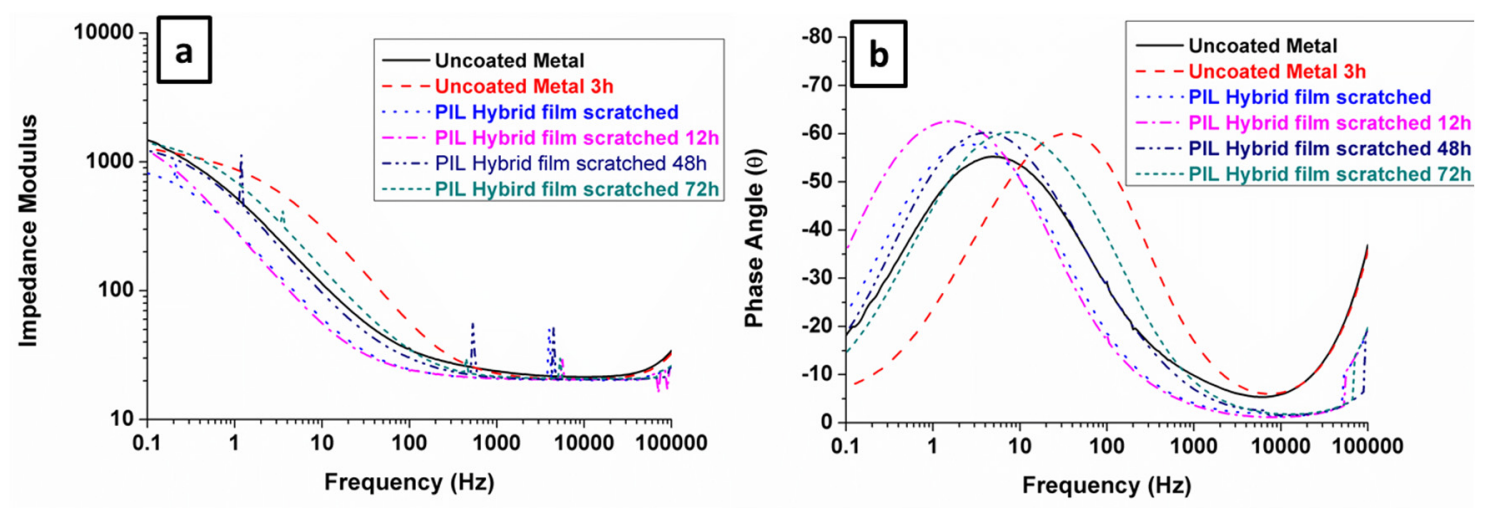

Figure 11. (a) Bode plot; (b) phase angle of the impedance of a scratched PIL hybrid film/SNAP coated sample over $72 \mathrm{~h}$ compared with uncoated metal samples.

\section{Conclusions}

We have successfully prepared the novel MPTS/PIL hybrid anticorrosive film for multilayer coating and investigated the chemical structure and thermal properties of the PIL hybrid film. Thermal analysis of MPTS/PIL hybrid film showed the high thermal stability of the PIL hybrid film with higher $T_{\mathrm{g}}$ due to the strong dual network formation of thiol-ene and sol-gel reaction. Further study using electrochemical investigation shows that PIL hybrid film offers significant protection and passivation of the metal surface in addition to imparting anti-corrosion properties under damaged condition. These results show that these MPTS/PIL hybrid films are promising interfacial layer in anti-corrosion applications.

Acknowledgments: The authors gratefully acknowledge the financial support of the Australian Research Council's Linkage grant for carrying out this work and also the industry partner Wave rider Energy for financial support of this work. The authors would like to thank Martin Johnston of Flinders University for assistance with the solid state NMR.

Author Contributions: N.K.D and N.R.C contributed to develop the concept and designed the experiments along with M.T. M.T. synthesized the materials and performed the experiments. S.S. supported M.M. to perform the electrochemical characterization. M.T. analyzed the data in collaboration with N.R.C, N.K.D. and S.S.M.T. wrote the draft with support from N.K.D., N.R.C.; and the manuscript was finalized through contributions of all the authors. All authors have given approval to the final version of the manuscript.

Conflicts of Interest: The authors declare no conflict of interest.

\section{References}

1. Khramov, A.N.; Balbyshev, V.N.; Voevodin, N.N.; Donley, M.S. Nanostructured sol-gel derived conversion coatings based on epoxy- and amino-silanes. Prog. Org. Coat. 2003, 47, 207-213. [CrossRef]

2. Rahimi, H.; Mozaffarinia, R.; Hojjati Najafabadi, A. Corrosion and wear resistance characterization of environmentally friendly sol-gel hybrid nanocomposite coating on AA5083. J. Mater. Sci. Technol. 2013, 29, 603-608. [CrossRef] 
3. Rahimi, S.K.; Potrekar, R.; Dutta, N.K.; Choudhury, N.R. Anticorrosive interfacial coatings for metallic substrates. Surf. Innov. 2013, 1, 112-137. [CrossRef]

4. Vijayalakshmi, U.; Rajeswari, S. Synthesis and characterization of sol-gel derived glass-ceramic and its corrosion protection on 316L SS. J. Sol-Gel Sci. Technol. 2007, 43, 251-258. [CrossRef]

5. Zheludkevich, M.L.; Salvado, I.M.; Ferreira, M.G.S. Sol-gel coatings for corrosion protection of metals. J. Mater. Chem. 2005, 15, 5099-5111. [CrossRef]

6. He, X.; Shi, X. Self-repairing coating for corrosion protection of aluminum alloys. Prog. Org. Coat. 2009, 65, 37-43. [CrossRef]

7. Metroke, T.L.; Parkhill, R.L.; Knobbe, E.T. Passivation of metal alloys using sol-gel-derived materials-A review. Prog. Org. Coat. 2001, 41, 233-238. [CrossRef]

8. Sanchez, C.; de A. A. Soler-Illia, G.J.; Ribot, F.; Lalot, T.; Mayer, C.R.; Cabuil, V. Designed hybrid organic-inorganic nanocomposites from functional nanobuilding blocks. Chem. Mater. 2001, 13, 3061-3083. [CrossRef]

9. Kannan, A.G.; Choudhury, N.R.; Dutta, N.K. Synthesis and characterization of methacrylate phospho-silicate hybrid for thin film applications. Polymer 2007, 48, 7078-7086. [CrossRef]

10. Vreugdenhil, A.J.; Balbyshev, V.N.; Donley, M.S. Nanostructured silicon sol-gel surface treatments for Al 2024-T3 protection. J. Coat. Technol. 2001, 73, 35-43. [CrossRef]

11. Donley, M.S.; Mantz, R.A.; Khramov, A.N.; Balbyshev, V.N.; Kasten, L.S.; Gaspar, D.J. The self-assembled nanophase particle (SNAP) process: A nanoscience approach to coatings. Prog. Org. Coat. 2003, 47, 401-415. [CrossRef]

12. Sinapi, F.; Forget, L.; Delhalle, J.; Mekhalif, Z. Self-assembly of (3-mercaptopropyl)trimethoxysilane on polycrystalline zinc substrates towards corrosion protection. Appl. Surf. Sci. 2003, 464-471. [CrossRef]

13. Sinapi, F.; Delhalle, J.; Mekhalif, Z. XPS and electrochemical evaluation of two-dimensional organic films obtained by chemical modification of self-assembled monolayers of (3-mercaptopropyl)trimethoxysilane on copper surfaces. Mater. Sci. Eng. 2002, 22, 345-353. [CrossRef]

14. Bagiyan, G.A.; Koroleva, I.K.; Soroka, N.V.; Ufimtsev, A.V. Oxidation of thiol compounds by molecular oxygen in aqueous solutions. Russ. Chem. Bull. 2003, 52, 1135-1141. [CrossRef]

15. Yuan, J.; Antonietti, M. Poly(ionic liquid) latexes prepared by dispersion polymerization of ionic liquid monomers. Macromolecules 2011, 44, 744-750. [CrossRef]

16. Taghavikish, M.; Subianto, S.; Dutta, N.K.; Choudhury, N.R. Facile fabrication of polymerizable ionic liquid based-gel beads via thiol-ene chemistry. ACS Appl. Mater. Interfaces 2015, 7, 17298-17306. [CrossRef] [PubMed]

17. Tremont, R.; de Jesús-Cardona, H.; García-Orozco, J.; Castro, R.J.; Cabrera, C.R. 3-mercaptopropyltrimethoxysilane as a $\mathrm{Cu}$ corrosion inhibitor in KCL solution. J. Appl. Electrochem. 2000, 30, 737-743. [CrossRef]

18. Damia, C.; Sarda, S.; Deydier, E.; Sharrock, P. Study of two hydroxyapatite/poly(alkoxysilane) implant coatings. Surf. Coat. Technol. 2006, 201, 3008-3015. [CrossRef]

19. Piwoński, I.; Grobelny, J.; Cichomski, M.; Celichowski, G.; Rogowski, J. Investigation of 3-mercaptopropyltrimethoxysilane self-assembled monolayers on $\mathrm{Au}(111)$ surface. Appl. Surf. Sci. 2005, 242, 147-153. [CrossRef]

20. Bauer, F.; Ernst, H.; Decker, U.; Findeisen, M.; Gläsel, H.-J.; Langguth, H.; Hartmann, E.; Mehnert, R.; Peuker, C. Preparation of scratch and abrasion resistant polymeric nanocomposites by monomer grafting onto nanoparticles, 1 FTIR and multi-nuclear NMR spectroscopy to the characterization of methacryl grafting. Macromol. Chem. Phys. 2000, 201, 2654-2659. [CrossRef]

21. Xu, A.; Zhang, F.Z.; Jin, F.; Zhang, R.; Luo, B.; Zhang, T. The evaluation of coating performance by analyzing the intersection of bode plots. Int. J. Electrochem. Sci. 2014, 9, 5116-5125. 
22. Messaadia, L.; ID El mouden, O.; Anejjar, A.; Messali, M.; Salghi, R.; Benali, O.; Cherkaoui, O.; Lallam, A. Adsorption and corrosion inhibition of new synthesized pyridazinium-based ionic liquid on carbon steel in $0.5 \mathrm{M} \cdot \mathrm{H}_{2} \mathrm{SO}_{4}$. J. Mater. Environ. Sci. 2015, 6, 598-606.

23. Galio, A.F.; Lamaka, S.V.; Zheludkevich, M.L.; Dick, L.F.P.; Müller, I.L.; Ferreira, M.G.S. Inhibitor-doped sol-gel coatings for corrosion protection of magnesium alloy AZ31. Surf. Coat. Technol. 2010, 204, 1479-1486. [CrossRef]

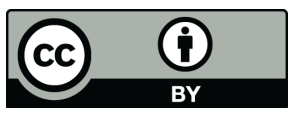

(C) 2016 by the authors; licensee MDPI, Basel, Switzerland. This article is an open access article distributed under the terms and conditions of the Creative Commons by Attribution (CC-BY) license (http://creativecommons.org/licenses/by/4.0/). 